\title{
CONTINUOUS FUNCTIONS TAKING EVERY VALUE A GIVEN NUMBER OF TIMES
}

\author{
ALEKSANDRA KWIATKOWSKA
}

\begin{abstract}
We give necessary and sufficient conditions on a function $f:[0,1] \rightarrow\{1,2, \ldots, \omega, \mathfrak{c}\}$ under which there exists a continuous function $F:[0,1] \rightarrow[0,1]$ such that for every $y \in[0,1]$, $\left|F^{-1}(y)\right|=f(y)$.
\end{abstract}

\section{Introduction}

A function $f:[0,1] \rightarrow\{1,2, \ldots, \omega, \mathfrak{c}\}$ will be called a (Banach) indicatrix if there exists a continuous function $F:[0,1] \rightarrow[0,1]$ such that $\left|F^{-1}(y)\right|=f(y)$ for each $y \in[0,1]$. We say that such a function $F$ is described by $f$. For each $y$, the set $F^{y}=\{x: F(x)=y\}$ will be called a section of $F$. In the paper we give a characterisation of indicatrices. Our result is a consequence of the Darboux Property and the Sierpiński-Mazurkiewicz Theorem, which in a special case says that for a continuous function $F:[0,1] \rightarrow[0,1]$, the set $F^{\mathfrak{c}}=\{y \in[0,1]$ : $\left.\left|F^{-1}(y)\right|=\mathfrak{c}\right\}$ is analytic. We also give an explicit construction of a function described by any given indicatrix. A general version of our result is given in Theorem 8 . In Theorem 1 we consider the case of $F$ such that $F(0)=0$ and $F(1)=1$.

The term indicatrix was introduced by Banach in [1]. He proved that the indicatrix of a continuous function is of Baire class 2 and $V(F)=\int_{0}^{1} f(y) d y$, where $F$ is a coninuous function described by $f$ and $V(F)$ is the variation of $F$.

A construction of a continuous $F$ such that $F^{\mathfrak{c}}$ is a given analytic set can be found in [5]. The case of $F^{\mathfrak{c}}=[0,1]$ can be found in [2] and [3]. Some partially results can also be found in [7]. A characterisation of indicatrices of Baire measurable and Lebesgue measurable functions is in [9] and of Marczewski measurable ones is given in [10]. In [8] there

1991 Mathematics Subject Classification. 03E15, 26A21.

Key words and phrases. indicatrix, continuous function, analytic set. 
are characterized (under Analytic Determinancy) indicatrices of Borel measurable functions.

The paper is organized as follows. In Chapter 2 in Theorem 1 we present a characterization of indicatrices of continuous functions $F$ such that $F(0)=0$ and $F(1)=1$ and prove that the conditions listed in this characterization are necessary. In Chapter 3 we prove that they are also sufficient for $F$ with countable sections. In Chapter 4 we deal with the general case and prove that the conditions listed in Theorem 1 are sufficient for $F$ which may have uncountable sections. In Chapter 5 we show that the assumption $F(0)=0$ and $F(1)=1$ may be dropped.

\section{The characterisation of indicatrices}

Define for $\kappa \in\{1,2, \ldots, \omega, \mathfrak{c}\} f^{\kappa}=\{y \in[0,1]: f(y)=\kappa\}, f^{\geq \kappa}=$ $\{y \in[0,1]: f(y) \geq \kappa\}$, and likewise define $f^{<\kappa}, f^{>\kappa}$, etc.

Theorem 1. A function $f:[0,1] \rightarrow\{1,2, \ldots, \omega, \mathfrak{c}\}$ is an indicatrix of a continuous function $F:[0,1] \rightarrow[0,1]$ such that $F(0)=0$ and $F(1)=1$ if and only if

(1) For all $y \in(0,1)$ such that $f(y) \in \omega$,

there exists $\varepsilon>0$ such that $y-\varepsilon<y_{1}<y<y_{2}<y+\varepsilon$ implies

$$
(*) \quad f\left(y_{1}\right)+f\left(y_{2}\right) \geq 2 f(y)
$$

and the equality can hold only if at least one (equivalently both) of $f\left(y_{1}\right)$ and $f\left(y_{2}\right)$ is odd.

Moreover, there exists $\varepsilon>0$ such that $0<y_{2}<\varepsilon$ implies $1+f\left(y_{2}\right) \geq$ $2 f(y)$ and $1-\varepsilon<y_{1}<1$ implies $f\left(y_{1}\right)+1 \geq 2 f(y)$.

(2) For all $y \in(0,1)$ such that $f(y)=\omega$ and for all but countably many $y$ such that $f(y)=\mathfrak{c}$,

for every $n \in \omega$ there exists $\varepsilon>0$ such that $y-\varepsilon<y_{1}<y<y_{2}<$ $y+\varepsilon$ implies

$$
(* *) \quad f\left(y_{1}\right)+f\left(y_{2}\right) \geq n .
$$

Moreover, for evry $n$ there exists $\varepsilon>0$ such that $0<y_{2}<\varepsilon$ implies $1+f\left(y_{2}\right) \geq 2 f(y)$ and $1-\varepsilon<y_{1}<1$ implies $f\left(y_{1}\right)+1 \geq 2 f(y)$.

(3) The set $f^{\mathfrak{c}}$ is analytic. 
Proof of necessity. (1) Assume that $0<y<1$ and $f(y)=n$. Let $a_{1}<a_{2}<\ldots<a_{n}$ be all points which $F$ sends to $y$. Consider intervals $\left[a_{k}, a_{k+1}\right], k=1,2, \ldots, n-1$ and choose $b_{k} \in\left(a_{k}, a_{k+1}\right)$. Let $\varepsilon=\min \left\{\left|F\left(b_{k}\right)-y\right|: k=1,2, \ldots, n-1\right\}$ and fix $y_{1} \in(y-\varepsilon, y)$ and $y_{2} \in(y, y+\varepsilon)$. It follows from the Darboux Property that in every interval $\left(0, a_{1}\right),\left(a_{k}, b_{k}\right),\left(b_{k}, a_{k+1}\right),\left(a_{n}, 1\right)$ either $y_{1}$ or $y_{2}$ is assumed at least once. There are $2 n$ intervals, therefore we get the inequality in $(*)$.

Now suppose that we have the equality in $\left(^{*}\right)$. Then in the intervals $\left(a_{k}, a_{k+1}\right), k=1,2, \ldots, n$, exactly one of the values $y_{1}$ and $y_{2}$ must be taken exactly twice, and in the intervals $\left(0, a_{1}\right),\left(a_{n}, 1\right)$ exactly once. If $f\left(y_{1}\right)$ and $f\left(y_{2}\right)$ were both even, then either $y_{1}$ or $y_{2}$ would be assumed in both of the intervals $\left(0, a_{1}\right),\left(a_{n}, 1\right)$. Then $F(0) \neq 0$ or $F(1) \neq 1$. We get a contradiction.

We similarly deal with the remaining part of $(1)$.

(2) Assume that $0<y<1, f(y) \in\{\omega, \mathfrak{c}\}$ and $F$ is not constant and equal $y$ in any interval. Fix $n \in \omega$. Choose $n$ points $a_{1}<a_{2}<\ldots<$ $a_{n}$ which $F$ sends to $y$. $F$ is not constant on any interval $\left[a_{i}, a_{i+1}\right]$. Similarly as before we get $\varepsilon>0$ such that $y-\varepsilon<y_{1}<y<y_{2}<y+\varepsilon$ implies $f\left(y_{1}\right)+f\left(y_{2}\right) \geq 2 n$, which gives $(* *)$.

We similarly deal with cases $y=0$ and $y=1$.

(3) This is a special case of the Sierpiński-Mazurkiewicz Theorem (a proof can be found in [6] in subchapter 4.3, in [4] or in [5]).

Note that in the proof of (1) and (2) it suffices that $F$ is a Darboux fuction. Therefore (1) and (2) characterise indicatices of Darboux functions $F$ satisfying $F^{\mathfrak{c}}=\emptyset, F(0)=0$ and $F(1)=1$.

Now we focus on the proof of sufficiency.

A function $f$ which satisfies conditions (1),(3) and satisfies (**) of (2) of Theorem 1 for all $y \in f^{\geq \omega}$ will be called a preindicatrix.

Remark 2. Without loss of generality we may drop the restriction "but countably many" from condition (2) of Theorem 1 and consider only preindicatrices.

Indeed, let $S$ be the countable set of $y \in[0,1]$ such that $f(y)=\mathfrak{c}$ and $f$ is bounded in some surrounding of $y$. 
Put for $y \in(0,1]$

$$
f\left(y^{-}\right)=\liminf _{z \rightarrow y^{-}} f(z)
$$

and for $y \in[0,1)$

$$
f\left(y^{+}\right)=\liminf _{z \rightarrow y^{+}} f(z),
$$

Let also $f\left(0^{-}\right)=1$ and $f\left(1^{+}\right)=1$

Note that $f\left(y^{+}\right), f\left(y^{-}\right) \leq \omega$.

For $y \in S$ put $f^{\prime}(y)=\min \left\{f\left(y^{-}\right), f\left(y^{+}\right)\right\}-1$ if $f\left(y^{+}\right)$and $f\left(y^{-}\right)$ are both even and equal and put $f^{\prime}(y)=\min \left\{f\left(y^{-}\right), f\left(y^{+}\right)\right\}$otherwise. Let $f^{\prime}(y)=f(y)$ outside $S$. Then $f^{\prime}$ is a preindicatrix.

Suppose we can find $F^{\prime}$ described by $f^{\prime}$ such that $F^{\prime}(0)=0$ and $F^{\prime}(1)=1$. At each level $y \in S$ inject into the graph of $F^{\prime}$ a small horizontal interval and shrink the resulting graph so that it has domain equal to $[0,1]$. In this way we get a function $F$ such that $\left|F^{-1}(y)\right|=\mathfrak{c}$ for $y \in S$ and $\left|F^{-1}(y)\right|=\left|F^{\prime-1}(y)\right|$ outside $S$. Therefore $F$ is described by $f$.

A preindicatrix whose range is contained in $\{1,2,3\}$ will be called a simple preindicatrix . For a simple preindicatrix $p$ any maximal interval of $p^{3}$ will be called interval of type three.

Remark 3. A function $p$ is a simple preindicatrix iff $p:[0,1] \rightarrow\{1,2,3\}$ and

a) $p^{3}$ is open;

b) if $p(y)=2$ then $y$ is an endpoint of some interval of type three.

Lemma 4. Assume that $f$ is a preindicatrix. Then there exist a preindicatrix $f^{\prime}$ and a simple preindicatrix $p$ such that for all $y \in[0,1]$,

$$
f(y)=(p(y)-1)+f^{\prime}(y) .
$$

(in the above equality if $f(y) \geq \omega$ put $f(y)=\infty$ ).

Proof. Set $p:[0,1] \rightarrow\{1,2,3\}$ as follows

$$
p(y)= \begin{cases}1 & \text { if } \quad f(y)=1 \\ 3 & \text { if } \exists_{\varepsilon>0} \forall_{z \in(y-\varepsilon, y+\varepsilon)} f(z)>2 \\ 2 & \text { otherwise }\end{cases}
$$


and let

$$
f^{\prime}(y)= \begin{cases}f(y)-p(y)+1 & \text { if } \quad f(y)<\omega \\ \infty & \text { if } \quad f(x) \geq \omega\end{cases}
$$

We show that the functions $p$ and $f^{\prime}$ are preindicatrices.

First we check a) and b) of Remark 3 for $p$.

Fix $y \in[0,1]$. Assume first that $p(y)=3$. Then for some neighbourhood $(y-\varepsilon, y+\varepsilon)$ of $y$ we have $f \geq 3$. In the same interval $p \equiv 3$. This implies that the set $\{y \in[0,1]: p(y)=3\}$ is open.

If $p(y)=2$ then $f(y) \geq 2$. Since $f$ is a preindicatrix, for some $\varepsilon>0$ $f \geq 3$ in $(y-\varepsilon, y)$ or in $(y, y+\varepsilon)$. Then $p \equiv 3$ in this neighbourhood, as desired.

Now we prove that $f^{\prime}$ is a preindicatrix. Fix $y \in(0,1)$.

Let first $p(y)=2$ and $f(y)<\omega$. We apply (1) of the already proved part of Theorem 1 to $f$ and fixed $y$ and take the resulting $\varepsilon>0$. If $f(y)=2$, then $f^{\prime}(y)=1$ and the required inequalities holds. Otherwise, without loss of generality there is $y_{0} \in(y-\varepsilon, y)$ such that $f\left(y_{0}\right) \in\{1,2\}$. Then if $y_{2} \in(y, y+\varepsilon)$ we obtain

$$
2 f(y) \leq f\left(y_{2}\right)+f\left(y_{0}\right)
$$

and equality can hold only if $f\left(y_{2}\right)$ and $f\left(y_{0}\right)$ are both odd, in particular when $f\left(y_{0}\right)=1$. Hence we get

$$
2 f(y) \leq 1+f\left(y_{2}\right)
$$

Therefore for $y_{1} \in(y-\varepsilon, y)$ and $y_{2} \in(y, y+\varepsilon)$ we get

$$
\begin{aligned}
& f^{\prime}\left(y_{1}\right)+f^{\prime}\left(y_{2}\right) \geq 1+\left(\left(1+f\left(y_{2}\right)\right)-p\left(y_{2}\right)\right) \geq 1+2 f(y)-p\left(y_{2}\right) \geq \\
& 1+2 f(y)-3 \geq 2(f(y)-p(y)+1) \geq 2 f^{\prime}(y) .
\end{aligned}
$$

If $f^{\prime}\left(y_{1}\right)$ and $f^{\prime}\left(y_{2}\right)$ are both even, then the first of above inequalities is strict, then

$$
f^{\prime}\left(y_{1}\right)+f^{\prime}\left(y_{2}\right)>2 f^{\prime}(y) .
$$

If $p(y)=1$ then $f(y)=1$. Thus also $f^{\prime}(y)=1$. Hence for this $y$ the demanded inequalities hold.

If $p(y)=3$ and $f(y) \in \omega$ then there exists a neighbourhood $(y-\varepsilon, y+$ $\varepsilon)$ of $y$ in which $p$ receives value three. Therefore for $x \in(y-\varepsilon, y+\varepsilon)$ 
we have $f^{\prime}(x)+2=f(x)$. Hence the demanded inequalities follow from the similar ones for $f$.

The remaining case, i.e. when $y \in\{0,1\}$ is much easier to check (we omit it).

Finally, if $f(y)=\infty$, then also $f^{\prime}(y)=\infty$. We apply (2) of the already proved part of Theorem 1 to $n+2$ and $f$ and get the required inequality for $f^{\prime}$. This completes the proof of the Lemma.

The following theorem will allow us to consider in the construction a sequence of simple preindicatrices instead of a preindicatrix.

Theorem 5. Assume that $f$ is a preindicatrix. Then there exist simple preindicatrices $p_{0} \geq p_{1} \geq p_{2} \geq \ldots$ such that for all $y \in[0,1]$

$$
\begin{aligned}
& f(y)=1+\sum_{i=0}^{\infty}\left(p_{i}(y)-1\right), \quad \text { if } f(y) \in \omega ; \\
& \infty=1+\sum_{i=0}^{\infty}\left(p_{i}(y)-1\right), \quad \text { if } f(y) \in\{\omega, \mathfrak{c}\} .
\end{aligned}
$$

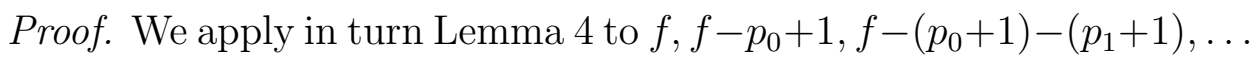
and obtain a sequence $p_{0}, p_{1}, p_{2}, \ldots$

Put

$$
f_{n}=p_{0}+\sum_{i=1}^{n}\left(p_{i}-1\right)
$$

The inequality $p_{n+1} \leq p_{n}$ follows from the definition of $p_{n}$.

Fix $y \in[0,1]$ such that $f(y)$ is finite. For $(1)$ it is enough to notice that for some $m \in \omega$

$$
f_{0}(y)<f_{1}(y)<\ldots<f_{m-1}(y)<f_{m}(y)=f_{m+1}(y)=\ldots=f(y)
$$

Fix $y \in[0,1]$ such that $f(y)=\infty$. For $(2)$ it is enough to notice that for each $n, p_{n}(y) \geq 2$.

\section{The construction with the assumption $f^{\mathfrak{c}}=\emptyset$}

We start with some preliminary notation. 
Let $\vec{R}$ be one of the diagonals in a rectangle $R=[a, b] \times[c, d]$. For $e=0,1,2$, consider rectangles $\left[a+e \frac{b-a}{3}, a+(e+1) \frac{b-a}{3}\right] \times[c, d]$ with diagonals $\vec{R}^{e}$ as shown in Figure 1 and $q_{\vec{R}}=\vec{R}^{0} \cup \vec{R}^{1} \cup \vec{R}^{2}$. Let $\pi_{X}$ and $\pi_{Y}$ be the projections onto the $x$ and $y$ axes. Note that $\pi_{X} \vec{R}=[a, b]$, $\pi_{Y} \vec{R}=[c, d], \pi_{Y} \vec{R}^{0}=\left[a, a+\frac{b-a}{3}\right]$, etc.

For an open interval $I=\left(c^{\prime}, d^{\prime}\right)$ define

$$
\vec{R} \cdot I=\vec{R} \cap\left([a, b] \times\left[c^{\prime}, d^{\prime}\right]\right),
$$

and for a family $P$ of open intervals, let

$$
\vec{R} \cdot P=\{\vec{R} \cdot I: I \in P\} .
$$

For a diagonal $\vec{R}$ let

$$
R=\pi_{X} \vec{R} \times \pi_{Y} \vec{R}
$$

denote the rectangle from which $\vec{R}$ arises. If $\overrightarrow{\mathcal{R}}$ is a family of diagonals, let $\mathcal{R}$ be the corresponding family $\{R: \vec{R} \in \overrightarrow{\mathcal{R}}\}$ of rectangles.
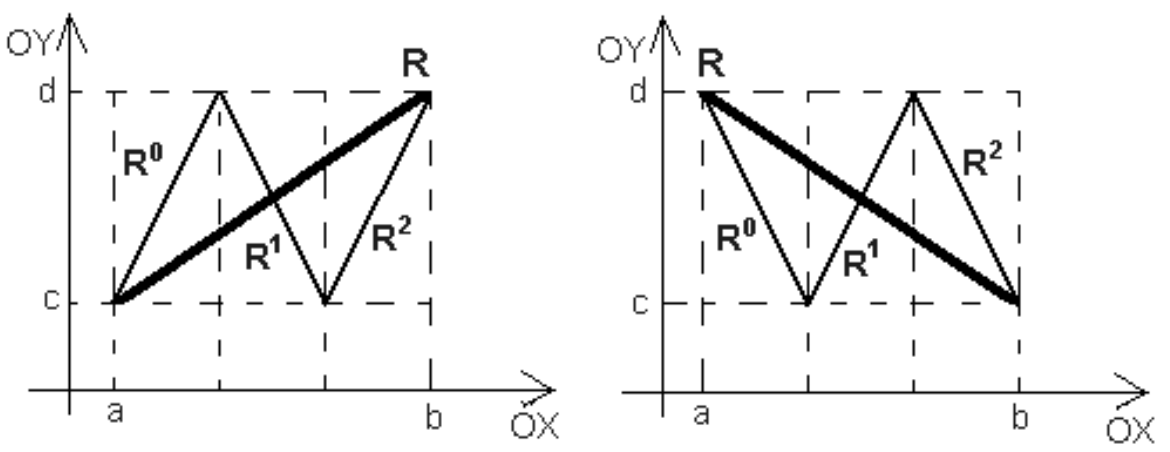

FiguRE 1. Graphs of $q_{\vec{R}}$.

We start with an idea of the construction. Fix a preindicatrix $f$. To the sequence of simple preindicatrices $p_{0}, p_{1}, p_{2}, \ldots$ (obtained from a preindicatrix $f$ via Theorem (5) we will assign families of open intervals $P_{0}, P_{1}, P_{2}, \ldots$, described below. Next, to each interval $I$ in $P=\bigcup_{i} P_{i}$ we assign a graph $q_{\vec{R}}$ for some diagonal $\vec{R}$ such that the closure of $I$ is equal to $\pi_{Y} \vec{R}$. All these parts glued together will form the required graph. 
First, let us take any simple preindicatrix $p$. Let $\left\langle y_{i}: i \in \omega\right\rangle$ be an enumeration of the endpoints of its intervals of type three. For every $y_{i}<y_{j}$, which are endpoints of the same interval of type three:

- if $p\left(y_{i}\right)=p\left(y_{j}\right)=1$, or if $p\left(y_{i}\right)=2$ and $p\left(y_{j}\right)=1$ and $y_{i}$ is the endpoint of two intervals of type three, we choose an increasing sequence $\left(y_{i, k}\right)_{k \in \mathbb{Z}}$ such that $\lim _{k \rightarrow \infty} y_{i, k}=y_{j}$ and $\lim _{k \rightarrow-\infty} y_{i, k}=y_{i}(\mathbb{Z}$ denotes the set of integers $)$.

- if $p\left(y_{i}\right)=1$ and $p\left(y_{j}\right)=2$, or if $p\left(y_{i}\right)=p\left(y_{j}\right)=2$ and $y_{i}$ is the endpoint of two intervals of type three, we choose an increasing sequence $\left(y_{i, k}\right)_{k<0}$ such that $\lim _{k \rightarrow-\infty} y_{i, k}=y_{i}$ and $y_{i,-1}=y_{j}$.

- if $p\left(y_{i}\right)=2$ and $p\left(y_{j}\right)=1$ and $y_{i}$ is the endpoint of exactly one interval of type three, we choose an increasing sequence $\left(y_{i, k}\right)_{k \geq 0}$ convergent to $y_{j}$, such that $y_{i, 0}=y_{i}$.

- in the remaining cases put $y_{j, 0}=y_{i}$ and $y_{j, 1}=y_{j}$.

Let $P$ be the set of all intervals $\left(y_{n, k}, y_{n, k+1}\right)$. We assign in this manner $P_{0}$ to $p_{0}, P_{1}$ to $p_{1}, \ldots$ Without loss of generality we assume that each interval in $P_{i+1}$ is has the length less then $\frac{1}{i+1}$, and is a subinterval of some interval in $P_{i}$ (if necessary, divide some interval $(a, b) \in P_{i}$ into some intervals $\left(a, c_{1}\right),\left(c_{1}, c_{2}\right),\left(c_{2}, c_{3}\right), \ldots,\left(c_{k}, b\right)$ for some $\left.k\right)$.

Now, assuming that $f^{\mathfrak{c}}=\emptyset$, we describe the construction. A graphical example illustrating the construction can be found after the description of the general construction at the end of Chapter 4.

Step 0.

We put $\overrightarrow{\mathcal{R}}_{0}=(i d \uparrow[0,1]) \cdot P_{0}$

Step $n+1$. We have from the nth step the family of diagonals $\overrightarrow{\mathcal{R}}_{n}$.

Define

$$
\overrightarrow{\mathcal{R}}_{n+1}=\bigcup_{\vec{R} \in \overrightarrow{\mathcal{R}}_{n}} \vec{R}^{0} \cdot P_{n}
$$

We define now a sequence of functions $F_{0}, F_{1}, F_{2} \ldots$ mapping $[0,1]$ onto $[0,1]$.

Let $F_{0}$ is $i d \uparrow[0,1], F_{1}$ is $F_{0}$ modified in each $\vec{R} \in \overrightarrow{\mathcal{R}}_{0}$ to $q_{\vec{R}}, F_{n+1}$ is $F_{n}$ modified in each $\vec{R} \in \overrightarrow{\mathcal{R}}_{n}$ to $q_{\vec{R}}$ (note that we modify only the left part of each $\vec{R}$ ). Finally we put $F=\lim _{n \rightarrow \infty} F_{n}$. 
Note that $F_{n+1}=F_{n}$ outside $\bigcup_{n} \mathcal{R}_{n}$ and that for each rectangle $R \in$ $\mathcal{R}_{n}$ the lenght of $\pi_{Y} R$ is less then $\frac{1}{n}$. This implies that the convergence is uniform and thereby $F$ is continuous. $F$ maps $[0,1]$ onto $[0,1], F(0)=$ 0 and $F(1)=1$.

Notice that each rectangle in $\mathcal{R}_{n+1}$ is contained in some rectangle in $\mathcal{R}_{n}$. A decreasing sequence $R_{0} \supseteq R_{1} \supseteq R_{2} \supseteq \ldots$ such that for each $n$ $R_{n} \in \mathcal{R}_{n}$ call a branch. Note that $\left|\cap R_{n}\right|=1$.

Now fix $y \in[0,1]$. We shall prove that $F$ receives every value $y$ exactly $f(y)$ times.

It is easy to prove by induction that $f_{n}$ defined as in the proof of Theorem 5 is the indicatrix of $F_{n+1}$.

If $f(y)=n$, then there exists such $m$, that for $k \geq m f_{k}(y)=$ $f(y)$. Clearly if $F_{m}^{-1}(y)=\left\{a_{1}, a_{2}, \ldots, a_{n}\right\}$ then $F_{k}^{-1}(y)=F^{-1}(y)=$ $\left\{a_{1}, a_{2}, \ldots, a_{n}\right\}$. Finally $\left|F^{-1}(y)\right|=n$.

Otherwise, if $f(y)=\omega$, we can either find exactly one branch $\left(R_{n}\right)_{n}$ such that $\pi_{Y}\left[\cap R_{n}\right]=y$, or we can find exactly two branches $\left(R_{n}\right)_{n}$ and $\left(R_{n}^{\prime}\right)_{n}$ such that $\pi_{Y}\left[\cap R_{n}\right]=\pi_{Y}\left[\cap R_{n}^{\prime}\right]=y$ (in this case for some $N \in \omega$ we have $R_{n}=R_{n}^{\prime}$ for $n<N$ and $R_{n} \neq R_{n}^{\prime}$ for $n \geq N$; moreover, for $n \geq N R_{n}$ are of the form $\left[a_{n}, b_{n}\right] \times\left[c_{n}, y\right]$ and $R_{n}^{\prime}$ are of the form $\left.\left[a_{n}^{\prime}, b_{n}^{\prime}\right] \times\left[y, d_{n}^{\prime}\right]\right)$.

In the first case, for each $n$, there are exactly two $x_{n}^{a}, x_{n}^{b}$ such that $\left(x_{n}^{a}, y\right),\left(x_{n}^{b}, y\right) \in R_{n} \backslash R_{n+1}$ and $F\left(x_{n}^{a}\right)=F\left(x_{n}^{b}\right)=y$. In the second case, if $n<N$ as above we have exactly two $x_{n}^{a}, x_{n}^{b}$ such that $\left(x_{n}^{a}, y\right),\left(x_{n}^{b}, y\right) \in$ $R_{n} \backslash R_{n+1}$ and $F\left(x_{n}^{a}\right)=F\left(x_{n}^{b}\right)=y$, and if $n \geq N$ there is exactly one $x_{n}^{a}$ such that $\left(x_{n}^{a}, y\right) \in R_{n} \backslash R_{n+1}$ and $F\left(x_{n}^{a}\right)=y$ and there is exactly one $x_{n}^{b}$ such that $\left(x_{n}^{b}, y\right) \in R_{n}^{\prime} \backslash R_{n+1}^{\prime}$ and $F\left(x_{n}^{b}\right)=y$ (note that for $n>N$ we have $\left.x_{n}^{a} \neq x_{n}^{b}\right)$.

This observation and the above remark that branches have one-point intersection gives us $\left|F^{-1}(y)\right|=\omega$.

Remark 6. If $r n g f$ is bounded then $F=F_{n}$ for some $n \in \omega$.

\section{The general construction}


For a sequence $\alpha \in 3^{\omega}$ write $W_{\alpha}$ for $\{n \in \omega: \alpha(n) \neq 0\}$ and let

$$
\mathbb{P}=\left\{\alpha \in 3^{\omega}:\left|W_{\alpha}\right|=\omega\right\}
$$

The set $f^{\mathfrak{c}}$ is analytic, so if it is nonempty, there exists a continuous mapping $\alpha \mapsto \alpha^{*}$ of $\mathbb{P}$ onto $f^{\mathfrak{c}}$ such that $W_{\alpha}=W_{\beta}$ implies $\alpha^{*}=\beta^{*}$. Such mapping can be obtained as a composition of a "projection" map of $\mathbb{P}$ onto $\mathbb{P}_{2}=\left\{\alpha \in 2^{\omega}: \exists_{n}^{\infty} \alpha(n) \neq 0\right\}$ changing all 2's in sequences into 1's, and a mapping from $\mathbb{P}_{2}$ onto $f^{\mathfrak{c}}$ (note that $\mathbb{P}_{2}$ is a copy of the Baire space $\left.\omega^{\omega}\right)$.

For a finite sequence $\tau \in 3^{<\omega}$ we put

$$
\tau^{*}=\left\{\alpha^{*}: \tau \subseteq \alpha, \alpha \in \mathbb{P}\right\}
$$

We will also use the notation introduced in the previous chapter.

As for the case $f^{\mathfrak{c}}=\emptyset$, to each interval in $P=\bigcup_{i} P_{i}$ we assign a graph $q_{\vec{R}}$ for some diagonal $\vec{R}$. All these parts glued together will form the required graph. We will use each of the intervals in $P$ exactly once. If $I, J \in P$ and $I \subseteq J$, then $\mathrm{J}$ will be used before $I$. In general we will not use at the step $i$ exactly intervals from $P_{i}, i \in \omega$, as it was when $f^{\mathfrak{c}}=\emptyset$.

Now we define families of diagonals $\vec{R}_{\tau}, \tau \in 3^{<\omega}$ inductively along $|\tau|$. To do this, at the step $n+1$ of induction, we also define families of intervals $P_{i}^{n+1}, i \in \omega$ and families of intervals $P_{i}^{n, k}, k, i \in \omega$. Each diagonal in every $\vec{R}_{\tau}$ will come from some (exactly one) interval in $P$ and two different diagonals will come from different intervals in $P$.

Step 0.

We put $\overrightarrow{\mathcal{R}}_{\langle\rangle}=(i d \uparrow[0,1]) \cdot P_{0}$ and we rename our families of intervals as follows

$$
P_{0}^{0}=P_{1}, P_{1}^{0}=P_{2}, \ldots, P_{i}^{0}=P_{i+1}, \ldots
$$

Step $n+1$. We have from the nth step: the families of intervals $P_{i}^{n}$, $i \in \omega$, and the families of diagonals $\overrightarrow{\mathcal{R}}_{\tau}, \tau \in 3^{n}$.

Let $\left\langle\tau_{k}: k<3^{n}\right\rangle$ be an enumeration of $3^{n}$. Inductively along $k$ for $0 \leq k<3^{n}$ we define families of diagonals $\vec{R}_{\tau},|\tau|=n+1, \tau=\tau_{k}^{\curlyvee} e, e=$ $0,1,2$; and families of intervals $P_{i}^{n, k}, i \in \omega$. 
At the k-th step we are given (defined at the (k-1)-th step) families of intervals $P_{i}^{n, k-1}, i \in \omega$ (if $k=0$ we let $P_{i}^{n,-1}=P_{i}^{n}$, which are also given at the beginning of this step).

Define

$$
\overrightarrow{\mathcal{R}}_{\tau_{k}^{\curvearrowright} 0}=\bigcup_{\vec{R} \in \overrightarrow{\mathcal{R}}_{\tau_{k}}} \vec{R}^{0} \cdot P_{0}^{n, k}
$$

For $e=1,2$ define

$$
\overrightarrow{\mathcal{R}}_{\tau_{k} e}=\bigcup\left\{\vec{R}^{e} \cdot P_{e}^{n, k}: \vec{R} \in \overrightarrow{\mathcal{R}}_{\tau_{k}}, \tau_{k}^{*} \cap \pi_{Y} \vec{R} \neq \emptyset\right\} .
$$

Also, to each $\vec{R} \in \overrightarrow{\mathcal{R}}_{\tau_{k} e}, e=0,1,2$, assign a label $\# R=e$.

Finally put for $i \in \omega$ and all intervals $I$

$I \in P_{i}^{n, k} \quad$ iff

- $I \in P_{i}^{n, k-1}$ and there is no $\vec{R} \in \overrightarrow{\mathcal{R}}_{\tau}$ such that $I \subseteq \pi_{Y} \vec{R}$, or

- $I \in P_{i+1}^{n, k-1}$ and $I \subseteq \pi_{Y} \vec{R}$ for some $\vec{R} \in \overrightarrow{\mathcal{R}}_{\tau}$ such that $\tau^{*} \cap \pi_{Y} \vec{R}=\emptyset$,

or

- $I \in P_{i+3}^{n, k-1}$ and $I \subseteq \pi_{Y} \vec{R}$ for some $\vec{R} \in \overrightarrow{\mathcal{R}}_{\tau}$ such that $\tau^{*} \cap \pi_{Y} \vec{R} \neq \emptyset$.

In other words: if $P_{i}^{\prime n, k-1}$ is $P_{i}^{n, k-1}$ without those intervals we have just used in the step $(n, k)$ and if for some $i$ there is $I \in P_{i+1}^{\prime n, k-1}$ such that $I$ is not contained in (equivalently does not intersect) any interval in $P_{i}^{\prime n, k-1}$, then we move $I$ to $P_{i}^{\prime n, k-1}$. We inductively iterate this operation. Finally, when for each $I \in P_{i+1}^{\prime n, k-1}$ there is $J \in P_{i}^{\prime n, k-1}$ such that $I \subseteq J$, we put $P_{i}^{n, k}=P_{i}^{\prime n, k-1}$.

This finishes the step $k$.

At $k=3^{n}-1$, let also $P_{i}^{n+1}=P_{i}^{n, k}, i \in \omega$.

This finishes step $n+1$.

For $n \in \omega$ let

$$
\mathcal{R}_{n}=\bigcup_{\tau \in 3^{n}} \mathcal{R}_{\tau}
$$

and

$$
\mathcal{R}_{\omega}=\bigcup_{n \in \omega} \mathcal{R}_{n}
$$

Note that $\mathcal{R}_{\omega}$ with inclusion becomes a tree. Accordingly, a decreasing sequence of rectangles $\left(R_{n}\right)_{n}, R_{n} \in \mathcal{R}_{n}$, will be called a branch and the sequence of correspnding labels $\left(\# R_{n+1}\right)_{n}$ will be called a label of this branch. Note that for any branch $\left(R_{n}\right)_{n}$ there is $(x, y)$ such 
that $\{(x, y)\}=\bigcap_{n \in \omega} R_{n}$. We will say that $\left(R_{n}\right)_{n}$ converges to $((x, y)$ $\left.\left(R_{n}\right)_{n} \rightarrow(x, y)\right)$.

Claim 1. For any $\alpha \in 3^{\omega}$ and $y \in[0,1]$ there are at most two branches $\left(R_{n}\right)_{n}$ that converge to $(x, y)$ for some $x$ and have label $\alpha$.

Proof: Assume that there is a branch $\left(R_{n}\right)_{n}$ with a label $\alpha$ such that $\left(R_{n}\right)_{n} \rightarrow(x, y)$ for some $x$. Each $R_{n}$ is assigned to some interval $J_{n}$, namely $J_{n}=\pi_{Y}\left[R_{n}\right]$ (there is only one such assignment). These intervals form a subsequence of some decreasing sequence $\left(I_{k}\right)_{k}$ such that $I_{k} \in P_{k}$ and $y \in I_{k}, k \in \omega$. Note that there are at most two such sequences $\left(I_{k}\right)_{k}$. On the other hand, there is not more then one subsequence $\left(\widetilde{J}_{n}\right)_{n}$ of $\left(I_{k}\right)_{k}$ such that the sequence $\left(\widetilde{R}_{n}\right)_{n}$ of rectangles assigned to it form a branch of label $\alpha$.

Claim 2. Suppose $\left(R_{n}\right)_{n}$ is a branch with label $\alpha$. Then if $W_{\alpha}$ is infinite then $\left(R_{n}\right)_{n}$ converges to $\left(x, \alpha^{*}\right)$ for some $x$.

Proof: For each $n \geq 1$ we have $R_{n} \in \mathcal{R}_{\alpha \mid n}$ and $R_{n+1} \in \mathcal{R}_{\alpha \mid n^{\wedge} \alpha(n)}$. If $n \in W_{\alpha}$ then $\alpha(n) \in\{1,2\}$ and therefore, because $R_{n+1} \subseteq R_{n}, R_{n+1}$ witness that $(\alpha \mid n)^{*} \cap \pi_{Y} R_{n} \neq \emptyset$. It contains $\beta_{n}^{*}$ for some $\beta_{n} \supseteq \alpha \mid n$. Now $\lim _{n \in W_{\alpha}} \beta_{n}=\alpha$, so by continuity $\lim _{n \in W_{\alpha}} \beta_{n}^{*}=\alpha^{*}$. Since also $\lim _{n \in W_{\alpha}} \beta_{n}^{*} \in \bigcap_{n \in W_{\alpha}} \pi_{Y} R_{n}=\bigcap_{n \in \omega} \pi_{Y} R_{n}$, then $\bigcap_{n \in \omega} \pi_{Y} R_{n}=\left\{\alpha^{*}\right\}$ and we are done.

We define now a sequence of functions $F_{0}, F_{1}, F_{2} \ldots$ mapping $[0,1]$ onto $[0,1]$. as follows:

$F_{0}$ is $i d \uparrow[0,1], F_{1}$ is $F_{0}$ modified in each $\vec{R} \in \overrightarrow{\mathcal{R}}_{0}$ to $q_{R}, F_{n+1}$ is $F_{n}$ modified in each $\vec{R} \in \overrightarrow{\mathcal{R}}_{n}$ to $q_{R}$ (an example of such $F_{2}$ can be found in Example 1). Finally we put $F=\lim _{n \rightarrow \infty} F_{n}$ and claim that this is our needed $F$. The convergence is uniform, hence $F$ is continuous, maps $[0,1]$ onto $[0,1]$, and $F(0)=0, F(1)=1$.

It remains to see that the sections of $F$ are as required.

Fix $y \in[0,1]$. Note that if $\left(R_{n}\right)_{n} \rightarrow(x, y)$ for some $x$ then $F(x)=y$. Moreover, there are countably many $x$ such that $F(x)=y$ and $(x, y)$ is not a limit of any branch. The reasoning is similar to that when we were proving that assuming $f^{\mathfrak{c}}=\emptyset f(a)=\omega$ iff $\left|F^{-1}(a)\right|=\omega$. Indeed, put $K_{0}=[0,1]^{2} \backslash \bigcup \mathcal{R}_{0}$ and $K_{n+1}=\bigcup \mathcal{R}_{n} \backslash \bigcup \mathcal{R}_{n+1}, n \in \omega$. Note that for every $n$ there are finitely many $x$ such that $(x, y) \in K_{n}$ and $F(x)=y$. 
It is enough to prove:

Lemma 7. Take $y \in[0,1]$.

(a) If $f(y)=n$ then there are exactly $n$ such $y$ that $F^{-1}(y)=n$.

(b) If $f(y)=\omega$ then there are countably many $x$ such that $(x, y)$ is the limit of some branch.

(c) If $f(y)=\mathfrak{c}$ then there is a perfect set $P$ such that for every $p \in P$ $(p, y)$ is the limit of some branch.

Proof. (a) As in the construction with $f^{\mathfrak{c}}=\emptyset$.

(b) Let $\left(R_{n}\right)_{n} \rightarrow(x, y)$ and $\alpha$ be the label of this branch. By Claim $2 W_{\alpha}$ is finite. There are countably many $\alpha$ such that $W_{\alpha}$ is finite. Claim 1 finishes the proof.

(c) Fix $\alpha$ such that $y=\alpha^{*}$. Put $T=\left\{\beta \in 3^{\omega}: W_{\beta}=W_{\alpha}\right\}$ and let $S=\{\beta\lceil n: \beta \in T, n \in \omega\}$ be the tree corresponding to $T$.

We shall construct inductively a family $\left(R_{\tau}\right)_{\tau \in S}$ such that $R_{\tau} \in \mathcal{R}_{\tau}$, if $\tau \subseteq \sigma$ implies $R_{\sigma} \subseteq R_{\tau}, y \in \pi_{Y} R_{\tau}$ and if $\beta \neq \beta^{\prime}$ implies $\bigcap_{n} R_{\beta \mid n} \neq$ $\bigcap_{n} R_{\beta^{\prime} \mid n}$. This will finish the proof of (c).

First fix a sequence of intervals $\left(I_{n}\right)_{n}$ such that $I_{n} \in P_{n}$ and $y \in I_{n}$ for each $n$. Every $R_{\tau}$ will be assigned to some interval in this sequence. As $R_{\emptyset}$ take the rectangle assigned to $I_{0}$ construted at the 0th step. Assume that we already have $R_{\tau}$ for some $\tau,|\tau|=n$. If $\tau^{\frown} 0 \in S$, take $R_{\tau^{\wedge} 0} \subseteq R_{\tau}$ constructed at the step $(\mathrm{n}+1)$. If $\tau^{\wedge} 1, \tau^{\wedge} 2 \in S$ take $R_{\tau^{\wedge} 1}$, $R_{\tau^{\wedge} 2} \subseteq R_{\tau}$ constructed at the step $(\mathrm{n}+1)$ (note that $\tau \in S$ implies $\tau^{*} \cap \pi_{Y} R_{\tau} \neq \emptyset$, which is witnessed by $\left.y\right)$. Note that it can happen that $R_{\tau^{\wedge} 1}$ and $R_{\tau^{\wedge} 2}$ have common edge. However, for all $e_{1}, e_{2} \in\{0,1,2\}$ we will have $R_{\tau^{\wedge} 1^{\wedge} e_{1}} \cap R_{\tau^{\wedge} 2^{\prec} e_{2}} \neq \emptyset$, whenever $R_{\tau^{\wedge} 2^{\prec} e_{1}}, R_{\tau^{\wedge} 2^{\prec} e_{2}} \in S$. In this manner we accomplish the last required condition on $\left(R_{\tau}\right)_{\tau}$.

This ends our proof that conditions listed in Theorem 1 are also sufficient.

Example 1. Let $f$ be a preindicatrix such that $f^{\mathfrak{c}} \neq \emptyset$.

Let $p_{0}=3$ in $(0,1)$ and $p_{0}(y)=2$ if $y \in\{0,1\}$.

Let $p_{1}=3$ in $\left(0, \frac{3}{4}\right), p_{1}(y)=2$ if $y \in\left\{0, \frac{3}{4}\right\}$ and $p_{1}=1$ in $\left(\frac{3}{4}, 1\right]$. 
Let $p_{2}=3$ in $\left(0, \frac{1}{2}\right), p_{2}(0)=1, p_{2}\left(\frac{1}{2}\right)=2$ and $p_{2}=1$ in $\left(\frac{1}{2}, 1\right]$.

Finally, let $p_{3}=3$ in $\left(\frac{1}{4}, \frac{1}{2}\right)$ and $p_{3}(y)=1$ for the remaining $y$.

The sketch of graph of $F_{2}$ is shown in Figure 2

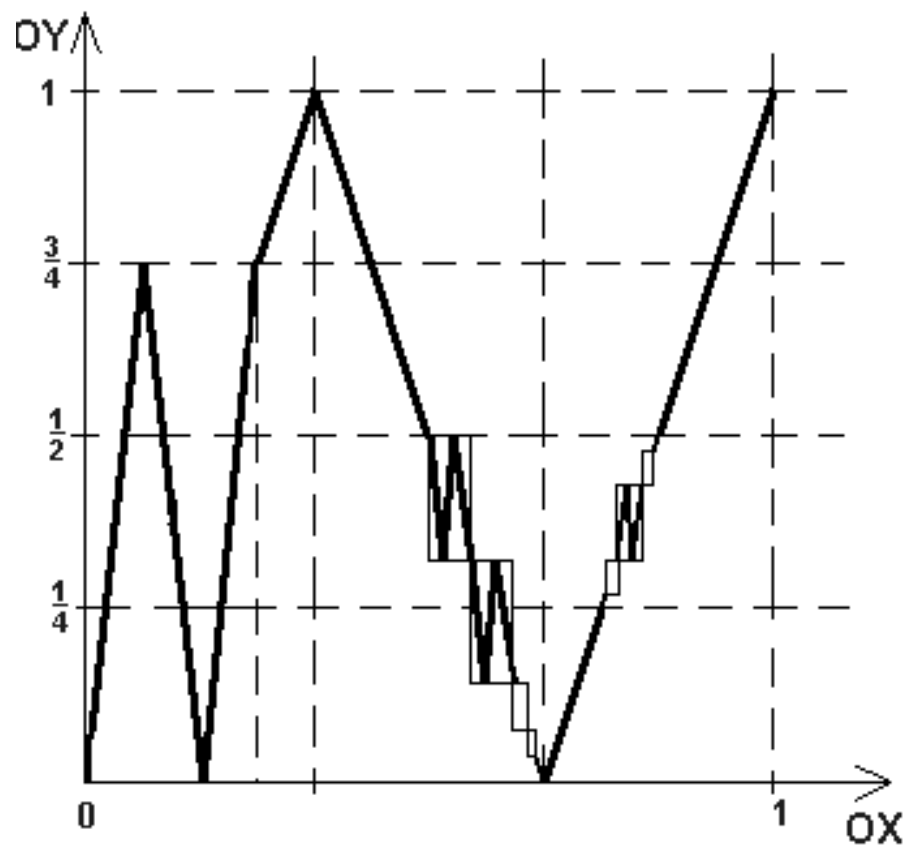

FiguRE 2. Sketch of graph of $F_{2}$.

\section{The general characterisation of indicatrices}

In the theorem below we present a general form of our result.

Theorem 8. $f$ is an indicatrix of a continuous function $F:[0,1] \rightarrow$ $[0,1]$ such that $F(0)=a$ and $F(1)=b$ for some $a \leq b$, if and only if:

(1) If $f(y) \in \omega$ and $y \in[0, a) \cup(b, 1]$ then there exists $\varepsilon>0$ such that for any $y_{1} \in(y-\varepsilon, y)$ and $y_{2} \in(y, y+\varepsilon)$ we have $f\left(y_{1}\right)+f\left(y_{2}\right) \geq$ $2 f(y)$ and if both of $f\left(y_{1}\right)$ and $f\left(y_{2}\right)$ are odd then $f\left(y_{1}\right)+f\left(y_{2}\right)>$ $2 f(y)$.

(2) If $f(y) \in \omega$ and $y \in(a, b)$ then there exists $\varepsilon>0$ such that for any $y_{1} \in(y-\varepsilon, y)$ and $y_{2} \in(y, y+\varepsilon)$ we have $f\left(y_{1}\right)+$ $f\left(y_{2}\right) \geq 2 f(y)$ and if both of $f\left(y_{1}\right)$ and $f\left(y_{2}\right)$ are even then $f\left(y_{1}\right)+f\left(y_{2}\right)>2 f(y)$. 
(3) If $f(y) \in \omega$ and $y=a=b$, then there exists $\varepsilon>0$ such that for any $y_{1} \in(y-\varepsilon, y)$ and $y_{2} \in(y, y+\varepsilon)$ we have $f\left(y_{1}\right)+$ $f\left(y_{2}\right) \geq 2(f(y)-1)$ and if both of $f(x)$ and $f(y)$ are odd then $f\left(y_{1}\right)+f\left(y_{2}\right)>2(f(y)-1)$.

(4) For all $y \in(0,1)$ such that $f(y)=\omega$ and for all but countably many $y$ such that $f(y)=\mathfrak{c}$,

for every $n \in \omega$ there exists $\varepsilon>0$ such that for any $y_{1} \in$ $(y-\varepsilon, y)$ and $y_{2} \in(y, y+\varepsilon)$ we have $f\left(y_{1}\right)+f\left(y_{2}\right) \geq n$.

(5) The set $f^{\mathfrak{c}}$ is analytic.

If $y=0$ or $y=1$ we put in the above inequalities $f\left(y_{1}\right)=1$ and $f\left(y_{2}\right)=1$ respectively (and we also omit " $y_{1} \in(y-\varepsilon, y)$ " and " $y_{2} \in$ $(y, y+\varepsilon) "$ respectively).

Proof. We start with the proof of necessity. Take $F$ as in the assumptions. Consider $\widetilde{F}$ such that $\widetilde{F} \uparrow\left[0, \frac{1}{3}\right]$ and $\widetilde{F} \uparrow\left[\frac{2}{3}, 1\right]$ are linear functions linking $(0,0)$ with $\left(\frac{1}{3}, a\right)$ and $\left(\frac{2}{3}, b\right)$ with $(1,1)$ respectively, and its graph over $\left[\frac{1}{3}, \frac{2}{3}\right]$ is a shrunk copy of $F$. Let $\tilde{f}$ describes $\widetilde{F}$. $\tilde{f}$ satisfies the conditions of Theorem 1. Now it can easily be checked that $f$ which describes $F$ satisfies the listed conditions.

Now we prove the sufficiency. Consider $\hat{f}$ such that $\hat{f}(y)=f(y)-1$ for $y \in(0, a) \cup(b, 1)$ and if $a \neq b$ also for $y \in\{a, b\}, \hat{f}(y)=f(y)-2$ if $y=a=b$, and $\hat{f}(y)=f(y)$ for other $y$. Notice that $\hat{f}$ satisfies the conditions of Theorem 1. Let $\widehat{F}$ such that $\widehat{F}(0)=0$ and $\widehat{F}(1)=1$ be described by $\hat{f}$. Let $F$ be such that its graph over $\left[\frac{1}{3}, \frac{2}{3}\right]$ is a shrunk copy of $\widehat{F}$, and $F \uparrow\left[0, \frac{1}{3}\right]$ and $F \uparrow\left[\frac{2}{3}, 1\right]$ are linear functions linking points $(0, a),\left(\frac{1}{3}, 0\right)$ and $\left(\frac{2}{3}, 1\right),(1, b)$ respectively. $F$ is described by $f$.

Finally note that $f$ is an indicatrix of $F$ such that $F(0)=a$ and $F(1)=b$ for some $a \geq b$ if and only if it is an indicatrix of $G$ such that for $x \in[0,1]$ we have $G(x)=F(1-x)$. Such a $G$ satisfies $G(0) \leq G(1)$.

Acknowledgments. I would like to thank E. Damek and J. Pawlikowski for their help in improving the exposition of the paper and to Szymon Glab for many remarks concerning the exposition. I also 
would like to thank M. Balcerzak, for a valuable review of an earlier version of the paper.

\section{REFERENCES}

[1] S. Banach Sur les lignes rectifiables et les surfaces dont l'aire est finie, Fund. Math. 7 (1925) pp. 225236

[2] A. Bruckner Differentation of Real Functions, CRM Monograph Series, Volume 5 [2nd edition], Providence, Rhode Island, 1994.

[3] J. Gillis Note on a conjecture of Erdös, Quart.J.Math.Oxford 10 (1939).

[4] A.S. Kechris Classical Descriptive Set Theory, Springer-Verlag, New York, 1995.

[5] Mazurkiewicz, Sierpiński Sur un probleme concernant les fonctions continues, Fund. Math. 6 (1924), 161-169.

[6] S.M. Srivastava A Course on Borel Sets, Springer-Verlag, New York, 1998

[7] K. Ciesielski, R.G. Gibson, T. Natkaniec $\kappa$-to-1 Darboux-like functions, Real Anal. Exchange 23 (1997-98), 671-687.

[8] A. Komisarski, H. Michalewski, P. Milewski Functions equivalent to Borel measurable ones, Preprint.

[9] M. Morayne, Cz. Ryll-Nardzewski Functions equivalent to Lebesgue measurable ones, Bull. Polish Acad. Sci. Math. 47 (1999), no.3, 263-265.

[10] M. Kysiak Some remarks on indicatrices of measurable functions, Bull. Polish Acad. Sci. Math. 53 (2005), 281-284.

Institute of Mathematics, University of Wroclaw, Plac GrunwalDZKI 2/4, 50-384 Wroclaw, Poland

E-mail address: ola_171@wp.pl 